\title{
A New Native Mass Spectrometry Platform Identifies Inhibitors of the HSP90 - HOP Protein-Protein Interaction
}

\author{
Clinton G. L. Veale, ${ }^{\text {*a }}$ Maria Mateos-Jimenez, ${ }^{b}$ Michaelone C. Vaaltyn, ${ }^{c}$ Ronel Müller, ${ }^{a}$ \\ Matodzi P. Makhubu, ${ }^{\mathrm{c}}$ Mahama Alhassan, ${ }^{\mathrm{a}}$ Beatriz G. de la Torre, ${ }^{\mathrm{d}}$ Fernando Albericio, ${ }^{\mathrm{a}} \mathrm{C}$. \\ Logan Mackay, ${ }^{b}$ Adrienne L. Edkins ${ }^{c}$ and David J. Clarke*b
}

\footnotetext{
a. FLAIR Research Fellow, School of Chemistry and Physics, Pietermaritzburg Campus, University of KwaZulu-Natal, Private Bag X01, Scottsville, 3209, South Africa.

b. EaStCHEM School of Chemistry, University of Edinburgh, Joseph Black Building, David Brewster Road, Edinburgh EH93FJ, UK

c. The Biomedical Biotechnology Research Unit (BioBRU), Department of Biochemistry and Microbiology, Rhodes University, Makhanda, South Africa.

d. KwaZulu-Natal Research Innovation and Sequencing Platform (KRISP), School of Laboratory Medicine and Medical Sciences, College of Health Sciences, University of KwaZulu-Natal, South Africa.
}

\begin{abstract}
Robust identification of inhibitors of Protein-Protein Interactions (PPIs) remains a significant drug discovery challenge, requiring multiple orthogonal analytical techniques. Herein we describe the development of a protein-truncated peptide model system that can act as a competent surrogate for the target HOP - HSP90 PPI in native mass spectrometry studies. The potential of this as a screening platform is demonstrated by identifying two new peptides capable of in vitro PPI disruption.
\end{abstract}

Traditional approaches to drug discovery have sought to disrupt critical biological processes through the direct inhibition of a well-defined subset of protein targets. ${ }^{1}$ However the majority of these protein targets, and their ensuing biological functions, are governed by highly specific interfacial associations between partner proteins. Targeting these Protein-Protein Interactions (PPIs) offers the opportunity to expand the volume of druggable chemical space, beyond the current tried and tested model. ${ }^{2-5}$ The most commonly cited challenge in identifying inhibitors of PPIs is the lack of molecular topography inherent to protein interfaces, which reduces opportunities for ligand binding. ${ }^{6,7}$ However, the development of robust assay platforms from which to identify PPI inhibitors, where difficulties in differentiating between genuine binding molecules and artefacts, as well as the relatively high false positive rates of fluorescent methodologies as a result of fluorescent or redox active small molecules, remains a substantial additional challenge.8,9 Therefore, the development of orthogonally operating methodologies which can measure changes to the binding affinity between two full-length proteins or between a full-length protein and a truncated peptide, acting 
as a protein-proxy, is required for the validation or indeed the identification of high quality hit PPI inhibitors. To this end, several common biophysical techniques including X-ray crystallography, nuclear magnetic resonance (NMR) spectroscopy, differential scanning fluorimetry, surface plasmon resonance and isothermal titration calorimetry, as well as non-native mass spectrometry based techniques have been employed in the development of PPI drug discovery platforms. ${ }^{10-12}$ However, it is common for these methods to suffer from relatively low throughput in addition to large sample consumption and extensive experimental set up. ${ }^{13}$

Conversely, native mass spectrometry (MS) provides a comparatively fast and extremely sensitive method for the analysis and direct monitoring of relatively weak non-covalent interactions of label-free protein assemblies and protein-ligand interactions in the gas phase. ${ }^{14,15}$ In addition, native MS analysis allows for the rapid evaluation of relative binding affinity and approximate dissociation constants, in addition to quantitative determination of binding stoichiometry of intact complexes and protein-ligand interactions. ${ }^{16,17}$ Furthermore, the orthogonal use of ion-mobility (IM), provides insight into conformation, structure, and stability of the complex, ${ }^{18,19}$ which previously, could only be observed through ${ }^{15} \mathrm{~N}$ labelled NMR experiments and X-ray co-crystallography. ${ }^{20}$ However, despite these advantages, no native MS based platforms for the identification of PPI inhibitors have been described. Given that PPIs offer an innovative means of inhibiting challenging drug targets, we turned our attention to Heat Shock Protein (HSP) 90. Despite their initial promise, traditionally derived $\mathrm{N}$ terminal ATP antagonists of HSP90 are associated with numerous limitations, preventing their clinical development. ${ }^{21}$ As such, disruption of the PPI between HSP90 and its $C$-terminal associating co-chaperones have been highlighted as viable targets to expand the HSP90 inhibition toolbox. ${ }^{22-24}$ One such co-chaperone HSP70-HSP90 organising protein (HOP), binds simultaneously to HSP70 and HSP90, and facilitates the transfer of partially folded client proteins thus mediating appropriate protein folding (Fig.1). Inhibition of this PPI, indirectly disrupts HSP90 mediated protein folding, without stimulating the compensatory transcriptional upregulation of HSP70, and is considered a promising target for cancer chemotherapy. ${ }^{25}$ In addition, recent evidence suggests that the HSP70-HOP-HSP90 ternary complex is required for proteasome assembly and efficient proteasomal-mediated protein turnover. ${ }^{26}$ The PPI interaction between HSP90 and HOP is mediated primarily by a series of salt bridges between a $C$-terminal MEEVD pentapeptide motif of HSP90 and a lysine rich region of the tetratricopeptide repeat 2A (TPR2A) domain of HOP. ${ }^{27}$ McAlpine and co-workers have recently reported the design of peptides which disrupt this PPI through binding to the 
MEEVD region of HSP90. ${ }^{28}$ We reasoned that an alternative means of disrupting the HOP-HSP90 interaction might be through identifying ligands which bind directly to HOP, an idea which has been explored by Regan and co-workers. ${ }^{29,30}$

Through a comprehensive study utilising acetylated analogues of MEEVD, Brinker et al. identified the interaction between the TPR2A domain of HOP and the MEEVD peptide as the fundamental contact for HOP-HSP90 association ${ }^{31}$ and as such represents a suitable proxy for studying the PPI. ${ }^{32}$ Further structural insight into this interaction was provided by an X-ray co-crystal between Ac-MEEVD-OH (1, Fig. 2) and TRP2A. ${ }^{33}$ Given the importance of this specific interaction to the final formation of the HOP-HSP90 PPI, we reasoned that it could prove a useful system to assess native IMMS as a tool for developing competent PPI models, suitable for use as PPI drug discovery platforms.

The use of native MS solution conditions and sample infusion by an automated nanoelectrospray robot allowed for chromatography-free rapid data acquisition times (20-60 second), suitable for sampling from multiwell plate formats (see supporting information). Using this system, native MS analysis of a 1:1 solution of TPR2A and $\mathbf{1}$, showed the formation of our desired interaction at an observed binding ratio of 1:0.7 (Fig. 3A and B).

IM analysis of the $[\mathrm{M}+8 \mathrm{H}]^{8+}$ ion of apo-TPR2A and the TPR2A-1 complex revealed similar arrival time distributions consisting of a major compact gas phase conformation (Fig. 3C). The calculated collisional cross section (CCS) values were in close agreement with the theoretical values determined by analysis of the available structures of the apo-TPR2A (pdb 2NC9) and TPR2A-1 complex (pdb 1ELR) (Fig. 3D). These data provided good evidence that the solution phase structure of the apo and ligand bound species was retained into the gas phase during native nESI ionisation, which is a key factor when attempting to identify genuine binding ligands. With our model in hand, we sought to determine whether it could be used as a tool to identify PPI inhibitors. Peptide 1, has traditionally been associated with weak disruption of the HOP. HSP90 PPI, ${ }^{31}$ however at our experimental concentration range, peptide 1 was incapable of disrupting the target PPI, in a solid phase ELISA assay (Fig. 4D).

Given the potential of tetrazoles as acid bioisosteres in drug discovery, ${ }^{34}$ we synthesized two Ac-MEEVD-OH analogues, Ac-MTrEVD-OH (2) and AcMETrVD-OH (3, Fig.2), in which one of the glutamic acid residues were substituted with the corresponding tetrazole containing non-canonical amino acid. Native MS analysis of TPR2A preincubated with either 2 (Fig. S1) or 3 (Fig. S2) revealed that both these 
tetrazole containing peptides bound to TPR2A with 1:1 stoichiometry at similar affinities as that observed for 1 . Native mass spectra obtained from a 1:1:1 buffered solution of TPR2A-1 co-incubated with either peptide $\mathbf{2}$ or $\mathbf{3}$ again produced the apo TPR2A and 1 bound species in addition to the species corresponding to the binding of peptide 2 or 3 (Fig. 4A and B). No evidence of a ternary complex, i.e. simultaneous peptide binding was observed, indicating that these peptides both competed for the same region of the target. The abundance ratios of each species indicated that in comparison to 1 , both tetrazole containing peptides preferentially bound to TPR2A, resulting in partial disruption of the TPR2A:MEEVD interface.

Ratiometric analysis of the signals corresponding to the three species observed in the mass spectra (Fig. 4C), revealed that in the absence of peptides 2 and $\mathbf{3}$ the relative abundance of apo TPR2A to TPR2A-1 existed at a ratio of $59 \%: 41 \%$. Introduction of peptide 2 and subsequent binding to TPR2A unsurprisingly reduced the relative abundance of apo TPR2A to $42 \%$, but had a far more pronounced effect on the TPR2A1 species, reducing this to $26 \%$. Similarly, peptide 3 was found to reduce apo-TPR2A abundance to $46 \%$, with the TPR2A-1 species further decreased to $20 \%$, representing a $51 \%$ change in abundance, thus strongly indicating that binding of peptide 3 to TPR2A occurred at via displacement of peptide 1.

In order to determine whether the disruption of 1 provided a competent model of PPI disruption, peptides $\mathbf{2}$ and $\mathbf{3}$ were subjected to an ELISA-based PPI solid phase assay, using the HSP90 $C$-terminal binding novobiocin, which weakly disrupts the HOPHSP90 PPI, as a control. ${ }^{35}$ Here both peptides were found to disrupt the PPI between the TPR2A and the HSP90 C-terminal in a dose-dependent manner (Fig. 4D). Interestingly, peptide $\mathbf{3}$, which was the most efficient peptide binding disruptor in the gas phase assay was also the most active in the solid phase assay, disrupting the target PPI at sub micromolar concentrations.

For a structural insight into the observed activity, we conducted an in silico assessment of our inhibitory peptides using the X-ray co-crystal structure or the TRR2A-1 complex (pdb 1ELR). ${ }^{33}$ In line with a previous in-depth in silico study, ${ }^{32}$ redocking of $\mathbf{1}$, followed by MM minimisation, placed the ligand in a similar binding mode to that solved for the co-crystal, where in addition to the Val4 residue of peptide 1 occupying a prominent hydrophobic pocket, the Met1 residue (Lys239), the side chain residue of Glu2 (Asn308, Arg305), the backbone carbonyl of Glu3 (Arg305), and both the terminal (Lys229, Asn233, Asn264) and side chain residue (Lys301, Gln298) of Asp5 formed a series of electrostatic interactions along the binding grove of TPR2A. Curiously 
however, the side chain residue of Glu3 was not found to participate in any electrostatic interactions (Fig. 5A). The highest scoring docking pose of peptide $\mathbf{3}$ showed that in contrast to the crystal structure, the bioisosteric tetrazole moiety interacted with Lys237. The preferential formation of this interaction, resulted in the ligand shifting position within the binding groove slightly, sacrificing its interaction with Asn308, whilst maintaining electrostatic interactions with the remaining residues as $\mathbf{1}$, albeit at different orientations (Fig. 5B). In silico assessment of peptide 2 showed that the tetrazole occupied a similar region to the corresponding glutamic acid of $\mathbf{1}$, thus reforming the interaction with Asn308. While the VD region of the peptide occupied a different region of the binding groove, these data also suggested that Glu3 interacted with Lys237, albeit to a lesser extent that peptide 3 (Fig. S3).

In addition to Lys238 and 239, Lys237 has been suggested to be a key residue for selective HOP-HSP90 interaction. ${ }^{36,37}$ Therefore, the capacity of peptides $\mathbf{2}$ and $\mathbf{3}$, to directly interact with this residue, with 1 seemingly less able to do so, may account for the peptide binding and PPI inhibitory activity, of these previously unreported tetrazole containing peptides, which provide new scaffolds for the development of HOP- HSP90 inhibitors, as well as tools exploring the biology of this important interaction.

To conclude, for the efficient exploitation of PPIs as tractable targets in drug discovery, the development of reliable orthogonal methods to identify or validate hit compounds is vital. We present here a proof of principle study demonstrating the potential of native MS based models for PPI inhibitor identification. Our methodology allowed us to structurally examine our protein-peptide model and its potential to act as a PPI surrogate. Furthermore, two rationally designed peptides containing one tetrazole sidechain residue, were found to disrupt the PPI model, which importantly, translated into in vitro PPI inhibition. Of note, the identification of genuine binders and PPI disruptors was conducted without the requirement of isotope labelling or tethering, without ligand interference. Given the advantages with respect to speed and sensitivity, as well as the relativity simple experimental set up required for native mass spectrometry, this approach provides an additional complimentary strategy to identity quality PPI inhibitors. Based on the inhibitory data obtained here, and the potential of this target, we are currently conducting an in depth medicinal chemistry study into the structural parameters required for selective PPI inhibition, with a view to further elucidating the role of this PPI in cancer pathogenesis and developing new therapies for difficult to treat cancers. 


\section{Conflicts of interest}

There are no conflicts to declare.

\section{Acknowledgments}

The authors acknowledge financial support from the National Research Foundation of South Africa (NRF, Grant Numbers 116305, 105829 and 127224), the South African Research Chairs Initiative of the Department of Science and Innovation (DSI) and NRF (Grant No 98566), the Royal Society-Newton Fund (Grant number NI160018), the BBSRC (Grant Number BB/R013993/1), Rhodes University, the University of KwaZuluNatal flagship initiative and Future Leaders - African Independent Research (FLAIR), a partnership between the African Academy of Sciences and the Royal Society that is funded by the UK Government as part of the Global Challenge Research Fund (GCRF). RM, MCV and MPM gratefully acknowledge support from the NRF. We thank the Centre for High Performance Computing (CHPC) for access to Schrodinger's modelling suite, Lynne Regan for the kind gift of the expression plasmid for TPR2A and Dr. Faye Cruickshank of the SIRCAMS mass spectrometry facility at the School of Chemistry, University of Edinburgh for technical support.

\section{References}

(1) Bull, S. C.; Doig, A. J. PLoS One 2015, 10, 1-44.

(2) Toogood, P. L. J. Med. Chem. 2002, 45, 1543-1558.

(3) Kuenemann, M. A.; Labbé, C. M.; Cerdan, A. H.; Sperandio, O. Sci. Rep. 2016, 6, 117.

(4) O. Villoutreix, B.; M. Labbe, C.; Lagorce, D.; Laconde, G.; Sperandio, O. Curr. Pharm. Des. 2012, 18, 4648-4667.

(5) Swart, T.; Khan, F. D.; Ntlantsana, A.; Laming, D.; Veale, C. G. L.; Przyborski, J. M.; Edkins, A. L.; Hoppe, H. C. Sci. Rep. 2020, 10, 4193.

(6) Wells, J. A.; McClendon, C. L. Nature 2007, 450, 1001-1009.

(7) Surade, S.; Blundell, T. L. Chem. Biol. 2012, 19, 42-50.

(8) Arkin, M. M. R.; Wells, J. A. Nat. Rev. Drug Discov. 2004, 3, 301-317.

(9) Taylor, I. R.; Dunyak, B. M.; Komiyama, T.; Shao, H.; Ran, X.; Assimon, V. A.; Kalyanaraman, C.; Rauch, J. N.; Jacobson, M. P.; Zuiderweg, E. R. P.; Gestwicki, J. E. J. Biol. Chem. 2018, 293, 4014-4025.

(10) Zhou, M.; Li, Q.; Wang, R. ChemMedChem 2016, 11, 738-756.

(11) Ferreira, L. G.; Oliva, G.; Andricopulo, A. D. Expert Opin. Drug Discov. 2016, 11, 957968. 
(12) Dailing, A.; Luchini, A.; Liotta, L. Expert Rev. Proteomics 2015, 12, 457-467.

(13) Chen, X.; Qin, S.; Chen, S.; Li, J.; Li, L.; Wang, Z.; Wang, Q.; Lin, J.; Yang, C.; Shui, W. Sci. Rep. 2015, 5, 8361.

(14) Fenn, J. B.; Mann, M.; Meng, C. K.; Wong, S. F.; Whitehouse, C. M. Science 2015, 246, 64-71.

(15) Clarke, D. J.; Campopiano, D. J. Analyst 2015, 140, 2679-2686.

(16) Hopper, J. T. S.; Robinson, C. V. In Proteomics for Biological Discovery; John Wiley \& Sons, Inc.: Hoboken, NJ, USA, 2019; pp 145-173.

(17) Guo, P.; Paul, A.; Kumar, A.; Harika, N. K.; Wang, S.; Farahat, A. A.; Boykin, D. W.; Wilson, W. D. Chem. Commun. 2017, 53, 10406-10409.

(18) Eyers, C. E.; Vonderach, M.; Ferries, S.; Jeacock, K.; Eyers, P. A. Curr. Opin. Chem. Biol. 2018, 42, 167-176.

(19) Veale, C. G. L.; Mateos Jimenez, M.; Mackay, C. L.; Clarke, D. J. Rapid Commun. Mass Spectrom. 2020, 34, e8570.

(20) Stojko, J.; Fieulaine, S.; Petiot-Bécard, S.; Van Dorsselaer, A.; Meinnel, T.; Giglione, C.; Cianférani, S. Analyst 2015, 140, 7234-7245.

(21) Wang, L.; Zhang, L.; Li, L.; Jiang, J.; Zheng, Z.; Shang, J.; Wang, C.; Chen, W.; Bao, Q.; Xu, X.; Jiang, Z.; Zhang, J.; You, Q. Sci. Adv. 2019, 5.

(22) Edkins, A. L. Top. Med. Chem. 2016, 19, 21-54.

(23) Buckton, L. K.; Wahyudi, H.; McAlpine, S. R. Chem. Commun. 2016, 52, 501-504.

(24) Chakraborty, A.; Edkins, A. L. Biochem. Biophys. Res. Commun. 2020.

(25) Cortajarena, A. L.; Yi, F.; Regan, L. ACS Chem. Biol. 2008, 3, 161-166.

(26) Bhattacharya, K.; Weidenauer, L.; Luengo, T. M.; Echeverría, P. C.; Bernasconi, L.; Wider, D.; Villemin, M.; Bauer, C.; Rüdiger, S. G. D.; Quadroni, M.; Picard, D. bioRxiv 2019, dx.doi.org/10.1101/562637.

(27) Onuoha, S. C.; Coulstock, E. T.; Grossmann, J. G.; Jackson, S. E. J. Mol. Biol. 2008, 379, 732-744.

(28) Rahimi, M. N.; McAlpine, S. R. Chem. Commun. 2019, 55, 846-849.

(29) Pimienta, G.; Herbert, K. M.; Regan, L. Mol. Pharm. 2011, 8, 2252-2261.

(30) Yi, F.; Regan, L. ACS Chem. Biol. 2008, 3, 645-654.

(31) Brinker, A.; Scheufler, C.; Von Der Mülbe, F.; Fleckenstein, B.; Herrmann, C.; Jung, G.; Moarefi, I.; Ulrich Hartl, F. J. Biol. Chem. 2002, 277, 19265-19275.

(32) Lapelosa, M. J. Chem. Theory Comput. 2017, 13, 4514-4523.

(33) Scheufler, C.; Brinker, A.; Bourenkov, G.; Pegoraro, S.; Moroder, L.; Bartunik, H.; Hartl, F. U.; Moarefi, I. Cell 2000, 101, 199-210.

(34) Allen, F.; Liebeschuetz, J. W.; Groom, C.; Bardwell, D. A.; Olsson, T. S. G.; Wood, P. A. J. Chem. Inf. Model. 2012, 52, 857-866. 
(35) Yun, B. G.; Huang, W.; Leach, N.; Hartson, S. D.; Matts, R. L. Biochemistry 2004, 43, 8217-8229.

(36) Daniel, S.; Bradley, G.; Longshaw, V. M.; Söti, C.; Csermely, P.; Blatch, G. L. Biochim. Biophys. Acta - Mol. Cell Res. 2008, 1783, 1003-1014.

(37) Muller, P.; Ruckova, E.; Halada, P.; Coates, P. J.; Hrstka, R.; Lane, D. P.; Vojtesek, B. Oncogene 2013, 32, 3101-3110. 
Figures

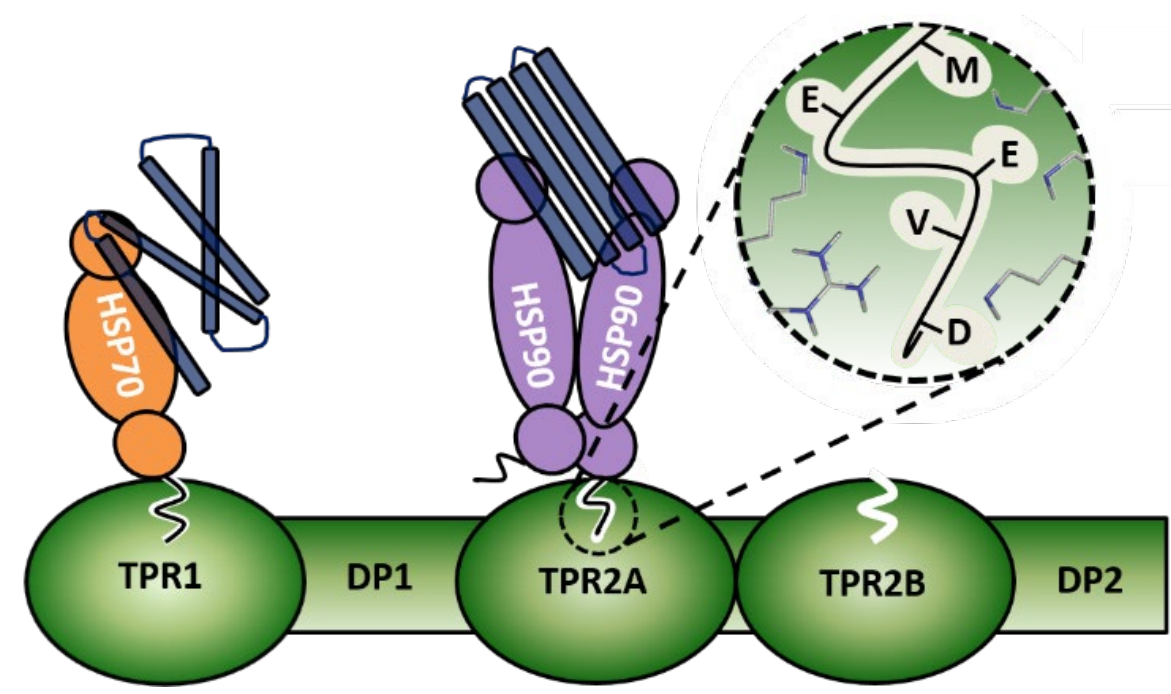

Figure 1. Schematic representation of the HSP70-HOP-HSP90 ternary chaperone complex, which mediates protein folding. The highlighted region, representing the PPI of interest in this study is controlled by the interaction of the acid rich HSP90 C-terminal MEEVD motif and the positively charged carboxylate clamp of the TPR2A domain of HOP. 

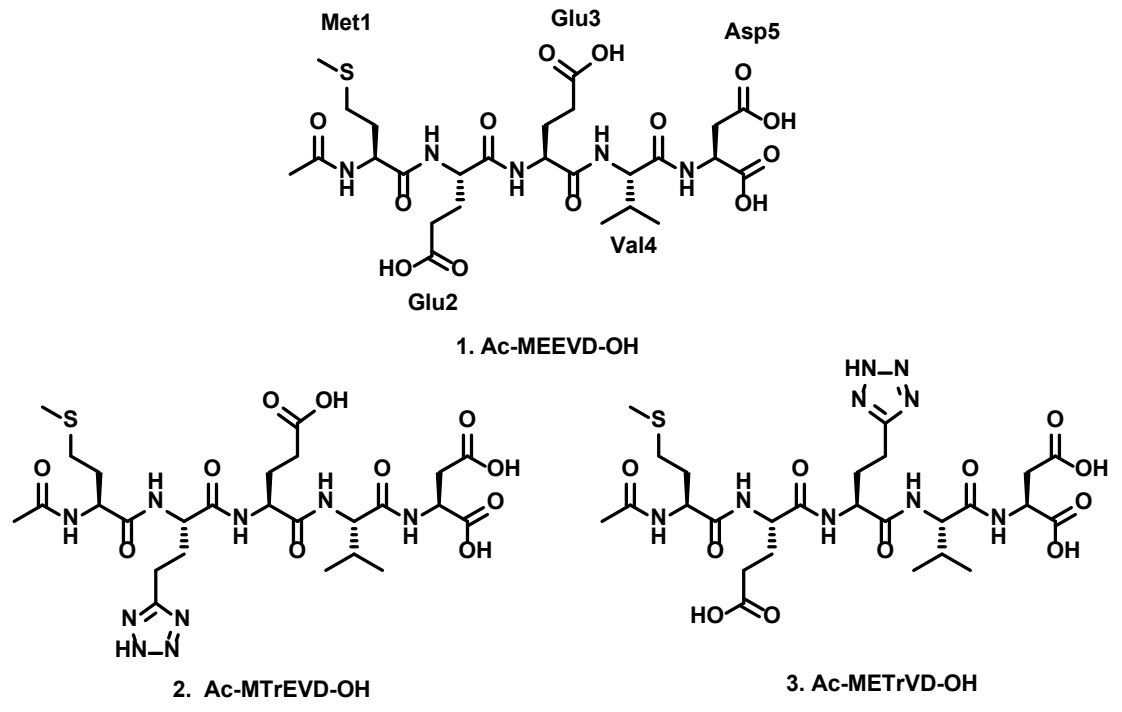

Figure 2. Pentapeptides used in this study 
A

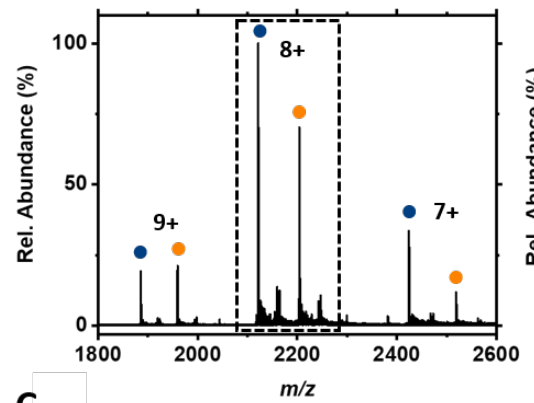

C

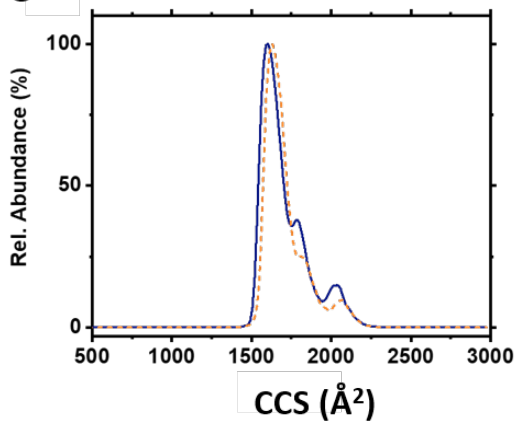

B

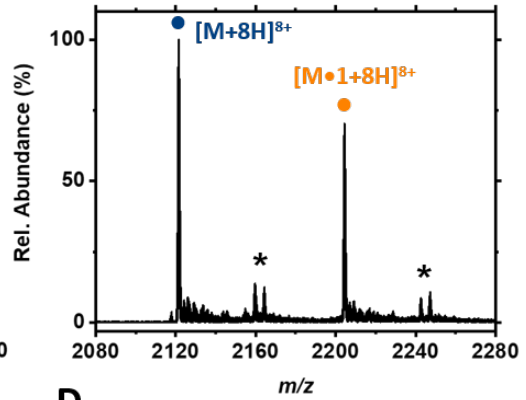

D

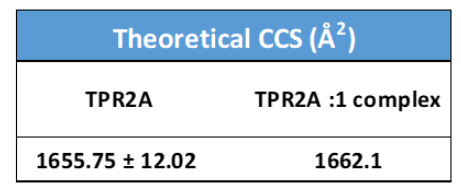

Experimental $\operatorname{CCS}\left(\AA^{2}\right)$

\begin{tabular}{|cc|}
\hline TPR2A & TPR2A : 1 complex \\
\hline $1603.55 \pm 161.1$ & $1630.62 \pm 151.7$ \\
\hline
\end{tabular}

Figure 3. A. Native mass spectrum of the 1:1 buffered solution of TPR2A and 1. B. Expanded region of the $8^{+}$charge state, showing the apo TPR2A (blue, $m / z 2121.5,[\mathrm{M}+8 \mathrm{H}]^{8+}$ ) and the TPR2A-1 complex (orange, $\mathrm{m} / \mathrm{z}$ 2204.4, $[\mathrm{M} \bullet 1+8 \mathrm{H}]^{8+}$ ). * protein impurities derived from protease cleavage during His-TPR2A purification. C. Ion mobility arrival time distribution of the 8+ charge state of TPR2A (blue solid line) and the TPR2A-AcMEEVD-OH complex (orange dashed line). D. CCS values of TPR2A and AcMEEVD-OH bound species determined theoretically (by analysis of 2NC9\# and 1ELR) and experimentally from ion mobility analysis. The errors quoted for experimental values represent full width and half max (FWHM) values. \#2NC9 is a solution structure, hence the margin of error in theoretical CCS 

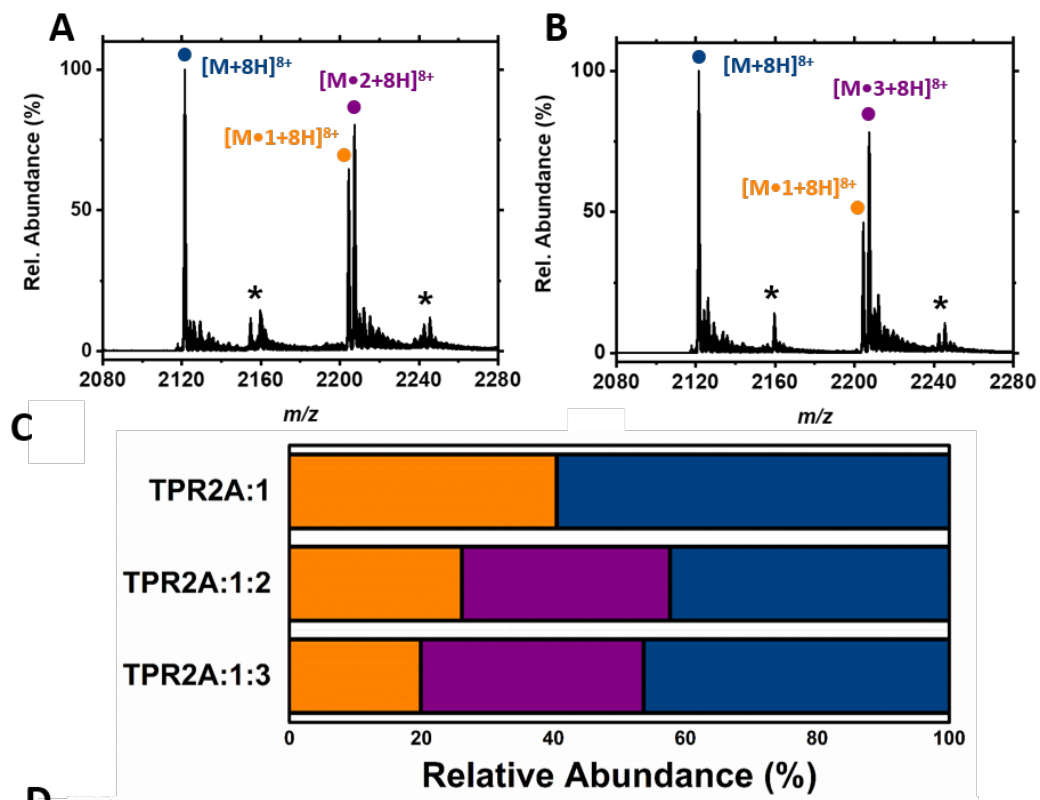

D

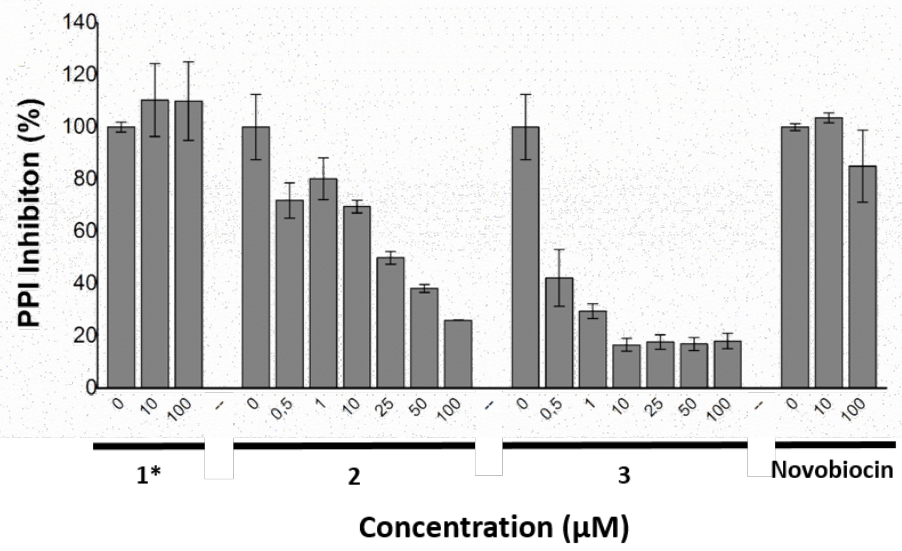

Figure 4: Expanded region of the $8^{+}$charge state, of the 1:1:1 mixture of TPR2A, 1 and 2 (A) or TPR2A, 1 and 3 (B) showing the apo TPR2A (blue $\mathrm{m} / \mathrm{z} 2121.5[\mathrm{M}+\mathrm{H}]^{8+}$ ) and the TPR2A-1 complex (orange, $\mathrm{m} / \mathrm{z} 2204.4[\mathrm{M}+\mathrm{H}]^{8+}$ ) and the TPR2A-2 or TPR2A-3 complexes (purple, $\mathrm{m} / \mathrm{z}$ $\left.2207.5[\mathrm{M}+\mathrm{H}]^{8+}\right)$. ${ }^{*}$ protein impurities derived from protease cleavage during His-TPR2A purification. C. Ratiometric analysis of the relative abundance of the MS signals corresponding to apo TPR2A (blue), the TPR2A-1 complex (orange) the TPR2A-2 or TPR2A-3 complexes (purple). The reduction in abundance of the TPR2A-1 complex, upon addition of peptides 2 and 3 suggest that these peptides inhibit binding of 1. D. Solid phase PPI ELISA assay, between the HOP TRP2A domain and the HSP90 C-terminal domain. Here peptides 2 (pIC $_{50}$ 5.1) and 3 ( $\mathrm{plC}_{50}$ 6.5) were both found to disrupt the PPI in a dose dependant manner. *AcMEEVD-OH assay was conducted previously on full length HOP. 

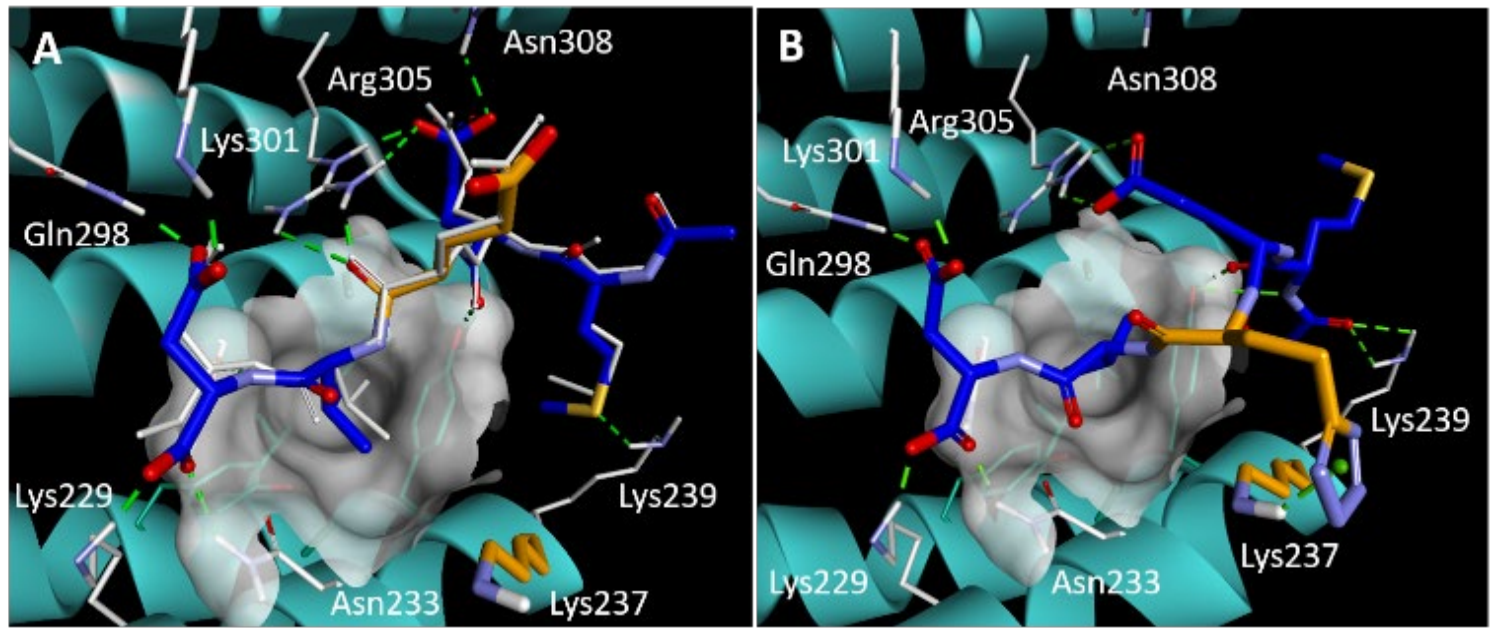

Figure 5A: Co-crystallised (white) and re-docked (blue/orange) binding pose of peptide 1 (RMSD 0.261), in the HSP90 recognition groove of the HOP-TPR2A domain (1ELR). White surface depicts the hydrophobic pocket. Glutamic acid 3 and Lys 237 (orange) do not interact. B: Lowest energy docked pose of peptide 3, indicating the formation of an electrostatic interaction between Lys237 and the tetrazole moiety, providing a structural basis for AcMEEVD-OH binding inhibition and PPI inhibition. 


\section{Supplementary information}

A

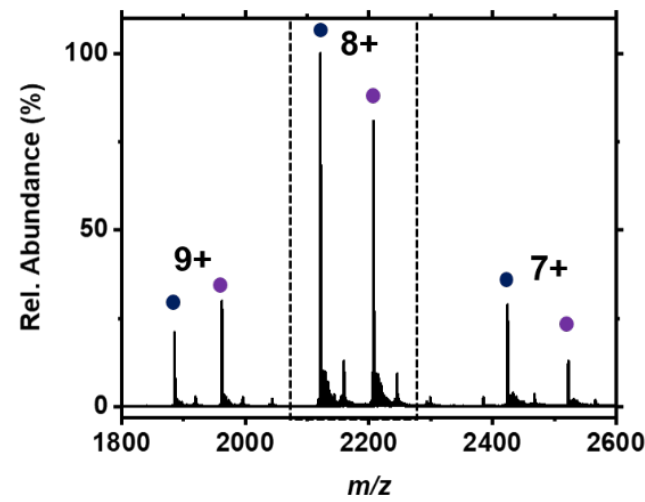

B

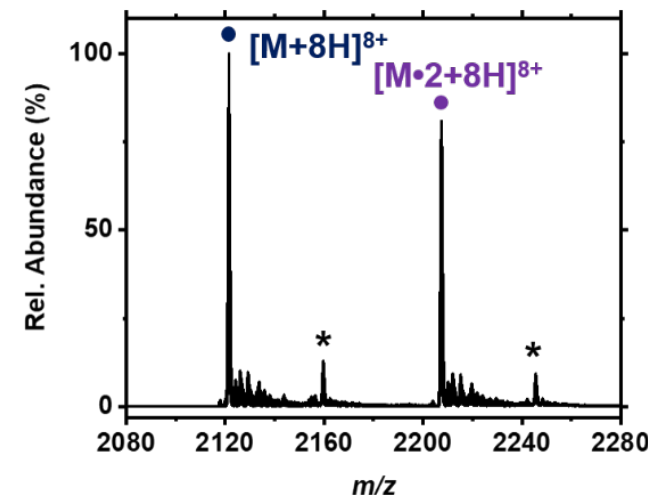

Figure S1A: Native MS spectrum of TPR2A and 2 (1:1) analysed by $\mathrm{nESI}$ from $50 \mathrm{mM}$ ammonium acetate. S1B: Expanded view of the 8+ charge state. Blue, TPR2A; purple, TPR2A-AcMTrEVD; * protein impurities derived from protease cleavage during His-TPR2A purification. 
A

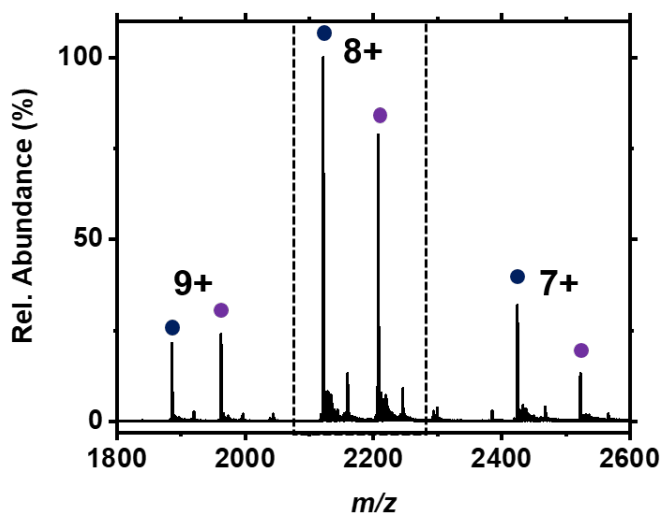

B

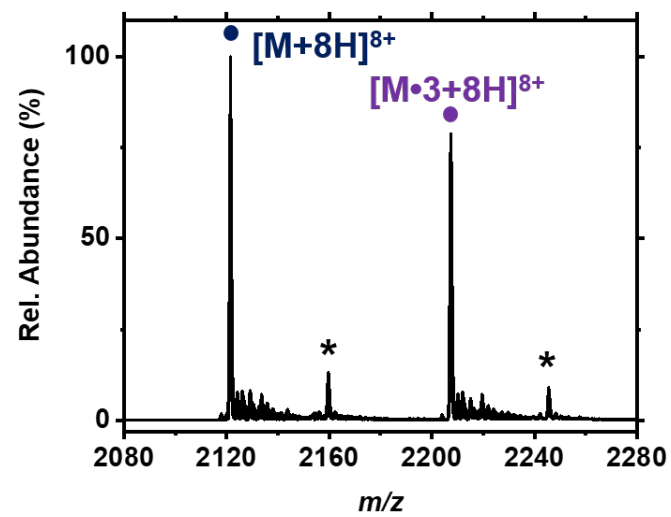

Fig S2A: Native MS spectrum of TPR2A and 3 (1:1) analysed by $\mathrm{nESI}$ from $50 \mathrm{mM}$ ammonium acetate. S2B: Expanded view of the 8+ charge state. Blue, TPR2A; purple, TPR2A-AcMETrVD; * protein impurities derived from protease cleavage during His-TPR2A purification. 


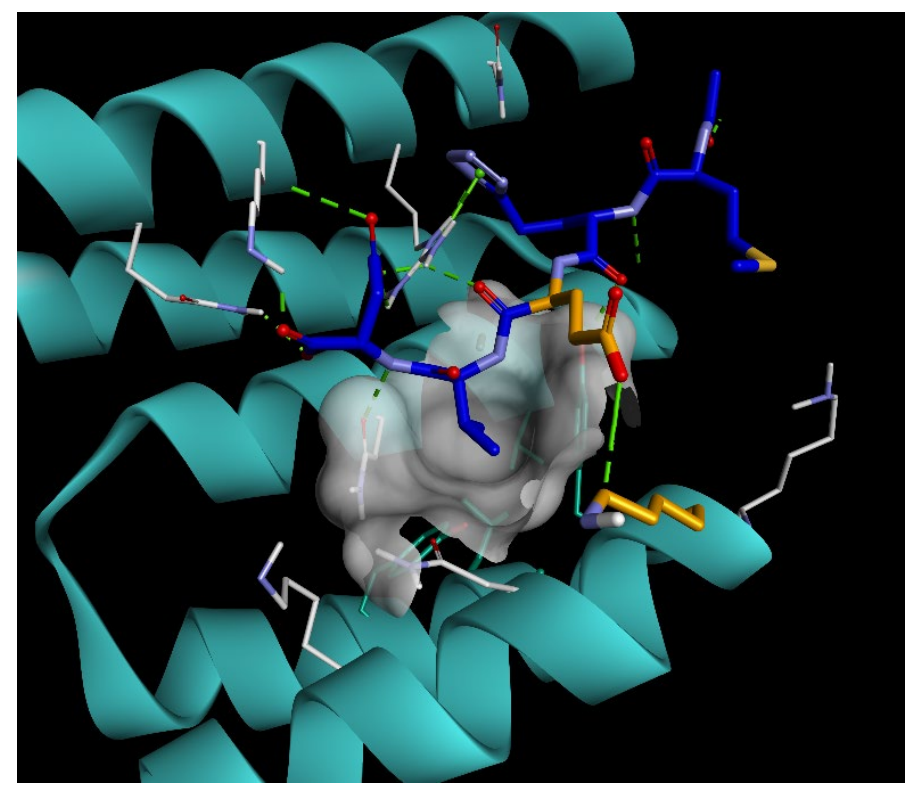

Figure S3: Lowest energy docked pose of peptide 2 


\section{Experimental Details.}

\section{Mass Spectrometry}

\section{Protein expression and purification}

His-tagged human TPR2A was expressed in E.coli and purified using Ni-charged IMAC column. Briefly, the TPR2A proEX-HTa plasmid was transformed into BL21(DE3) cells (New England Biolabs) and incubated in LB media containing ampicillin $(100 \mu \mathrm{g} / \mathrm{mL})$ at $37^{\circ} \mathrm{C}$ until the OD600 reading reached $0.6-0.7$. Then, protein expression was induced by adding IPTG 1 $\mathrm{mM}\left(37^{\circ} \mathrm{C}\right)$ for 3 hours. Cultures were harvested $\left(4000 \times \mathrm{g}, 4^{\circ} \mathrm{C}, 30 \mathrm{~min}\right)$ and stored frozen ($\left.20{ }^{\circ} \mathrm{C}\right)$. For protein purification, cell pellets were thawed and sonicated at $10 \mathrm{~A}(12 \times 30 \mathrm{~s}$ bursts) on ice. The cellular debris was harvested $\left(12000 \times \mathrm{g}, 4^{\circ} \mathrm{C}, 60 \mathrm{~min}\right)$. His-TPR2A was purified using a HisTrap HP column $1 \mathrm{~mL}$ (GE Healthcare Life Sciences) according to manufacturer's instructions. The His tag was cleaved with TEV protease at $4{ }^{\circ} \mathrm{C}$ overnight and the soluble TPR2A was subsequently purified by affinity chromatography. The accurate mass of the protein was confirmed by LC-MS.

\section{Sample Preparation for Native MS analysis}

Protein samples were buffer exchanged into $50 \mathrm{mM} \mathrm{NH}_{4} \mathrm{OAc}$ using Zeba Spin Desalting Column (Thermo Fisher Scientific) prior to MS analysis. The ligands were diluted in $50 \mathrm{mM}$ $\mathrm{NH}_{4} \mathrm{OAc}$ as required and preincubated with TPR2A $(5 \mu \mathrm{M})$ in 96 -well plates and held at $4{ }^{\circ} \mathrm{C}$ prior to MS analysis.

\section{Native MS/IM-MS}

Native MS and IM-MS data were obtained on both a Synapt-G2 Q-TOF (Waters) and a 12T SolariX 2XR FT-ICR (Bruker Daltonics). In both cases, ionisation was achieved using a NanoMate nESI infusion robot (TriVersa), sampling from a 96-well plate. Typically, a nanoelectrospay voltage of $1.55 \mathrm{kV}$ was used and backing pressure was adjusted to maintain stable electrospray. For IM-MS analysis on the Synapt platform, typical Native MS conditions included a source temperature of $60{ }^{\circ} \mathrm{C}$ and a backing pressure of $5.26 \mathrm{mbar}$ and nitrogen was used as the drift gas. MS data were processed using MassLynx v4.0 (Waters) and DriftScope v2.7 (Waters). Theoretical CCS values were calculated using IMPACT. ${ }^{1}$

For analysis on the FT-ICR platform a source temperature of $60^{\circ} \mathrm{C}$ was used and the source and transfer optics were tuned to maintain noncovalent complexes. Typically, spectra were acquired as the sum of 2001 MegaWord FID transients. DataAnalysis software (Bruker Daltonics) was used for the ratiometric analysis of different protein species. A mass list was generated using the FTMS algorithm ( $\mathrm{S} / \mathrm{N}$ threshold of 4 ). Then, the intensity of the most abundant isotopic peak from each of the three native charge states $(9+, 8+$ and $7+)$ was 
combined for each protein species. The summed abundance of for each species was used to determine the relative ratio of each of the TPR2A and TPR2A-peptide bound species.

\section{Peptide Synthesis}

Peptides were synthesized manually at $0.1 \mathrm{mmol}$ scale by $\mathrm{Fmoc} / \mathrm{tBu}$ protocol in a syringe fitted with a porous polyethylene disc and attached to a vacuum trap for easy filtration. The syntheses were carried out on 2-CTC resin (loading $0.86 \mathrm{mmol} / \mathrm{g}$ ) which was activated with $50 \%$ thionyl chloride in DCM for two hours. Then resin was washed properly with DCM and first coupling was done with Fmoc-Asp(tBu)-OH (3eq) in DCM in presence of $\mathrm{N}, \mathrm{N}$ diisopropylethylamine (DIEA) (10 eq) for two hours, then $\mathrm{MeOH}(50 \mu \mathrm{L})$ was added for capping the unreacted $\mathrm{Cl}$ groups for $30 \mathrm{~min}$ at room temperature. For Fmoc removal treatment with $20 \%$ piperidine in DMF for $7 \mathrm{~min}$ was done at each cycle. The remaining couplings were done with a 3-fold excess of Fmoc-amino acid, N, N'-diisopropylcarbodiimide (DIC) and OxymaPure in $1: 1: 1$ ratio for $45 \mathrm{~min}$. At the end of the chain elongation, peptides were acetylated at the end terminus using acetic anhydride (10eq), DIEA (20eq) in DMF for 45 minutes prior to full deprotection and cleavage with $\mathrm{TFA} / \mathrm{H}_{2} \mathrm{O} / \mathrm{TIS}$ (triisopropylsilane) (95:2.5:2.5 v/v, $90 \mathrm{~min}, \mathrm{RT}$ ). Peptides were precipitated by addition of chilled diethyl ether, taken up in water and lyophilized.

\section{Peptide analysis.}

Analytical HPLC was performed on an Agilent 1100 system using a Phenomenex Aeris ${ }^{\mathrm{TM}} \mathrm{C} 18$ $(3.6 \mu \mathrm{m}, 4.6 \times 150 \mathrm{~mm})$ column, with flow rate of $1.0 \mathrm{~mL} / \mathrm{min}$ and UV detection at $220 \mathrm{~nm}$. Chemstation software was used for data processing. Solvent $A$ was $0.1 \%(v / v)$ TFA in $\mathrm{H}_{2} \mathrm{O}$, solvent $\mathrm{B}$ was $0.1 \%(\mathrm{v} / \mathrm{v})$ TFA in $\mathrm{CH}_{3} \mathrm{CN}$. Elution was done with linear gradient 5 to $95 \%$ of solvent B into A over 15 min. Preparative HPLC was performed on Phenomenex C18 (21.2 × $250 \mathrm{~mm}, 10 \mu \mathrm{m}$ ) LC-ESI-MS was performed on a Thermo Scientific Dionex UltiMate 3000 using Phenomenex Aeris ${ }^{\mathrm{TM}} \mathrm{C} 18(3.6 \mu \mathrm{m}, 4.6 \times 150 \mathrm{~mm})$ column. Solvent A was $0.1 \%(\mathrm{v} / \mathrm{v})$ formic acid in $\mathrm{H}_{2} \mathrm{O}$, solvent $\mathrm{B}$ was $0.1 \%$ (v/v) formic acid in $\mathrm{CH}_{3} \mathrm{CN}$. 

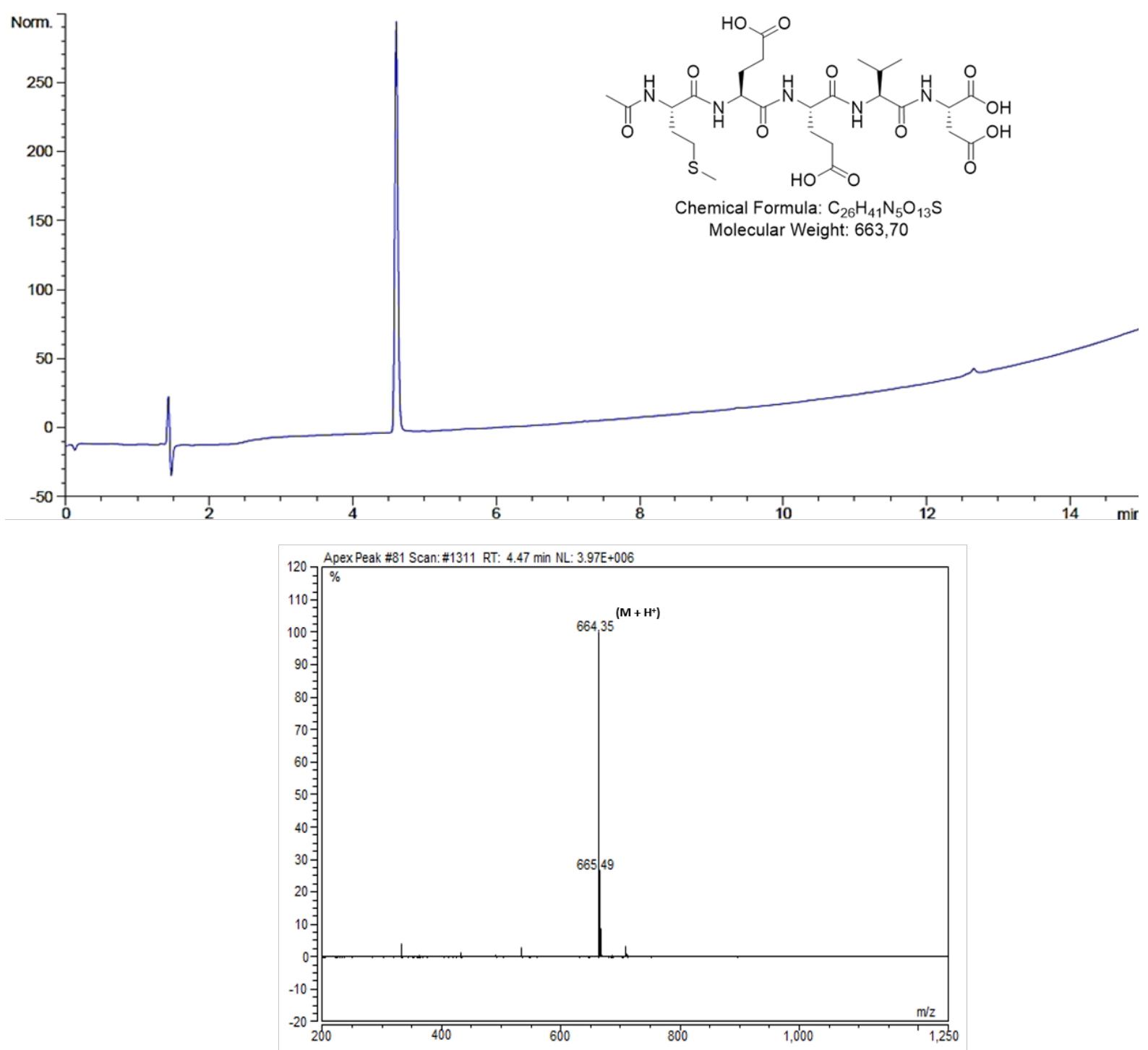

Fig S4 Top: Analytical HPLC trace of peptide 1, using a solvent gradient $5-95 \%$.

Compound purity determined to be $>99 \%$. Bottom: ESI Mass spectrum of peptide 1 corresponding with predicted mass of peptide 


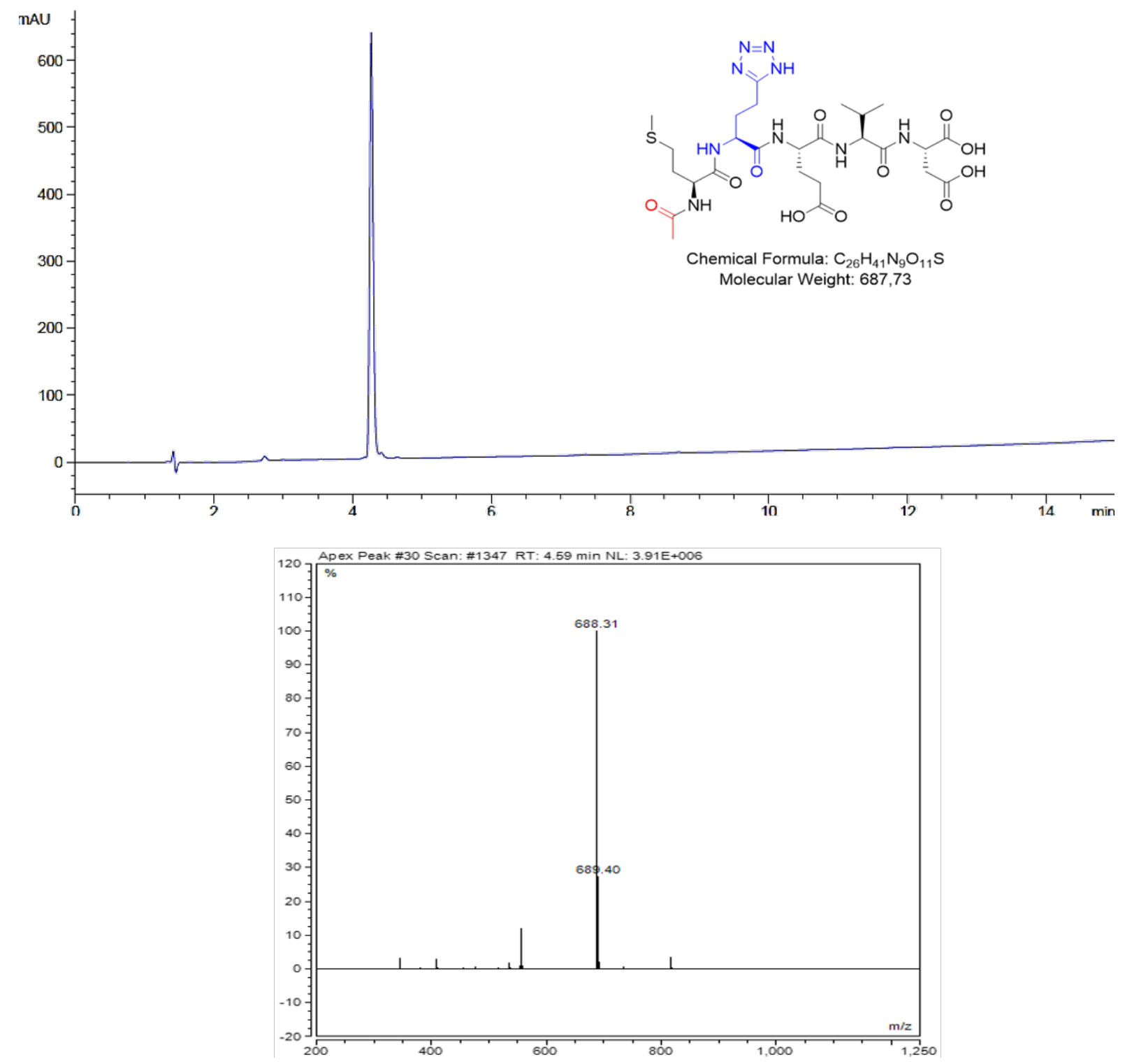

Fig S5 Top: Analytical HPLC trace of peptide 2, using a solvent gradient $10-60 \%$. Compound purity determined to be $>98 \%$. Bottom: ESI Mass spectrum of peptide 2 corresponding with predicted mass of peptide. 

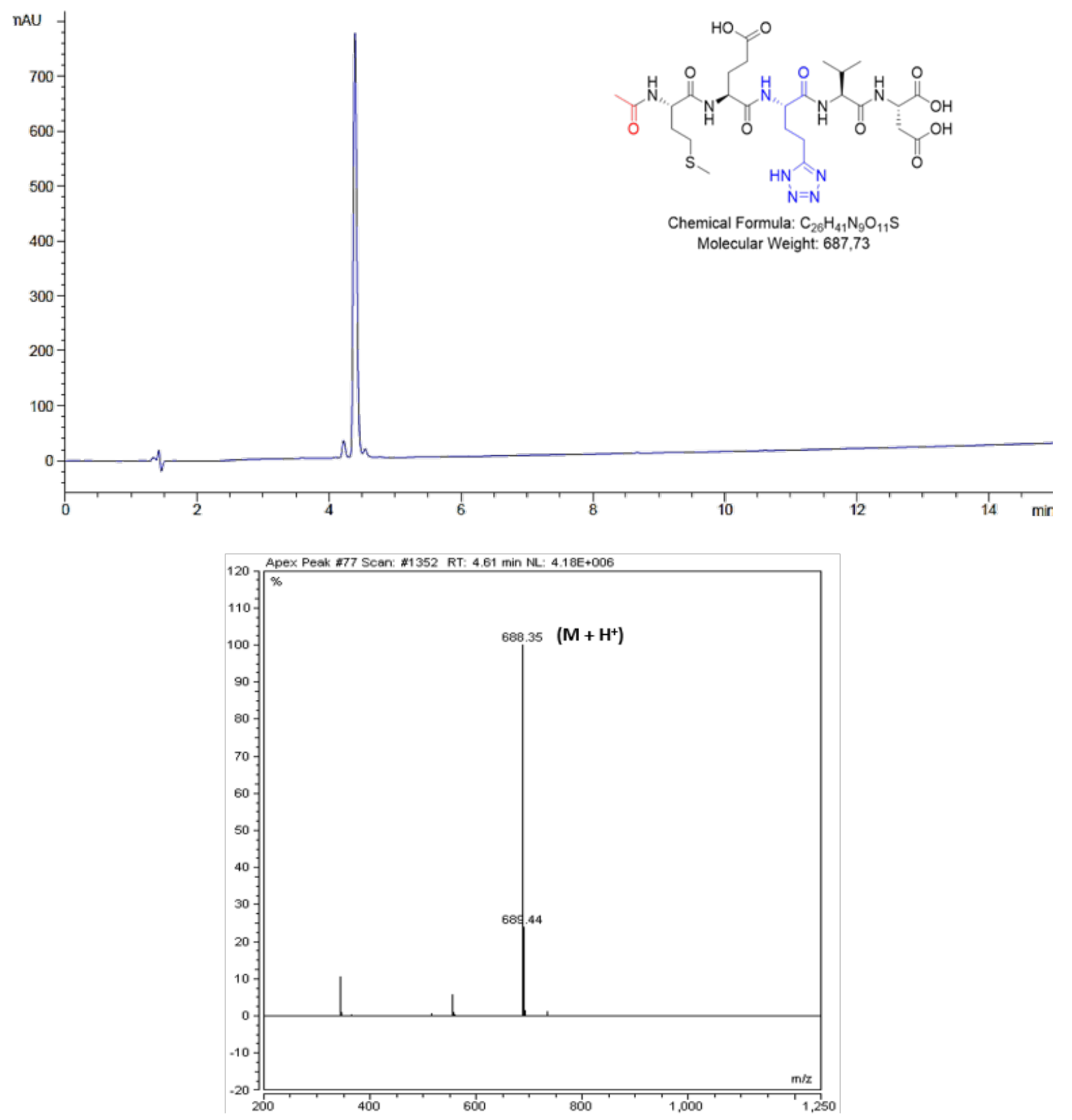

Fig S6 Top: Analytical HPLC trace of peptide 3, using a solvent gradient $10-60 \%$. Compound purity determined to be $>95 \%$. Bottom: ESI Mass spectrum of peptide 3 corresponding with predicted mass of peptide. 


\section{Biological assessment}

\section{Recombinant protein production and purification}

Recombinant proteins were produced in E.coli. GST was produced from the pGEX-4T-1 plasmid, while GST-Hsp90aC (residues 626-732 of Hsp90a) was produced from the pGEX4T3-Hsp90a-C (626-732) plasmid which was a gift from William Sessa (Addgene plasmid \#22483; http://n2t.net/addgene:22483; RRID:Addgene_22483). ${ }^{2}$ His-mSTI1 was produced from the pQE30-2000 plasmid. $^{3}$ The His-SUMO-TPR2A protein was produced from the pCA258-SUMO-TPR2A plasmid (encompassing residues 201 to 360 of human Hop). The pCA258 backbone was a gift of Matthias Mayer (ZMBH, Heidelberg, Germany). The murine (mSTI1) and human (Hop) versions of the protein share $98 \%$ amino acid identity. ${ }^{4}$ GSTtagged proteins and His-tagged proteins were purified from bacterial lysates by glutathione affinity chromatography or nickel-nitrilotriacetic acid affinity chromatography, respectively, according to established protocols. ${ }^{5}$

\section{Solid phase binding analysis for protein- protein interactions}

Interaction between GST-Hsp90C and either His-mSTI1 or His-SUMO-TPR2A was assessed as previously described. ${ }^{6}$ His-mSTI1 $(100 \mu \mathrm{g} / \mathrm{mL})$ or His-SUMO-TPR2A $(100 \mu \mathrm{g} / \mathrm{mL})$ were coated on a high-binding ELISA plate in buffer $\mathrm{A}$ [50 mM Tris- $\mathrm{HCl}, \mathrm{pH} 7.5,150 \mathrm{mM} \mathrm{NaCl}, 0.05$ $\%(v / v)$ Tween]) for $30 \mathrm{~min}$ at room temperature, followed by overnight at $4{ }^{\circ} \mathrm{C}$. Non-specific binding sites were blocked with 1 hour room temperature incubation with $3 \%(w / v)$ or $5 \%(w / v)$ BSA in Buffer A, for plates with His-SUMO-TPR2A and His-mSTI1, respectively. For the HismSTI1 plate, GST-Hsp90aC $(1 \mu \mathrm{M})$ in Buffer B (Buffer $A+0.1 \%[\mathrm{w} / \mathrm{v}] \mathrm{BSA})$ were incubated alone or with treatments (peptides or novobiocin) overnight at $4{ }^{\circ} \mathrm{C}$. For the His-SUMO-TPR2A plate, the peptides were incubated for 30 minutes at room temperature and 16 hours overnight at $4{ }^{\circ} \mathrm{C}$, after which GST-Hsp90aC $(1 \mu \mathrm{M})$ was added and incubated for 2 hours at room temperature. Thereafter, both sets of plates were washed thrice with $1 \%(\mathrm{w} / \mathrm{v}) \mathrm{BSA}$ in buffer $A$. The His-mSTI1 plate was incubated with rabbit anti-GST antibody (1 in 5000) in buffer A for $2 \mathrm{hrs}$ at room temperature. The His-SUMO-TPR2A plate was incubated with mouse antiGST primary antibody ( 1 in 1000 dilution) in buffer A for 16 hours at $25^{\circ} \mathrm{C}$.. Primary antibody incubation was followed by three washes in $1 \%(\mathrm{w} / \mathrm{v}) \mathrm{BSA}$ and incubation with speciesmatched secondary antibody ( 1 in 5000 dilution) for 2 hours at room temperature. After three washes with $1 \%(\mathrm{w} / \mathrm{v})$ BSA, HRP substrate [0.05 M phosphate-citrate buffer, $0.005 \%(\mathrm{v} / \mathrm{v})$ $\mathrm{H}_{2} \mathrm{O}_{2}, 1 \mathrm{mg} / \mathrm{mL} 3,3^{\prime}, 5,5^{\prime}$-Tetramethylbenzidine, $1 \% \mathrm{v} / \mathrm{v}$ DMSO] was added and incubated in the dark for at least 20 minutes at room temperature. The reaction was stopped with addition of $2 \mathrm{M} \mathrm{H}_{2} \mathrm{SO}_{4}$ and the absorbance read at $450 \mathrm{~nm}$. 


\section{In silico assessment}

Molecular mechanics simulations were performed using the Schrödinger Maestro software suite. ${ }^{7}$ The protein structure of interest, $1 \mathrm{ELR},{ }^{8}$ was downloaded from the Protein Data Bank. The protein was optimised at $\mathrm{pH} \mathrm{7,0}$ and bad contacts were removed using the protein preparation wizard. The protein was thereafter subjected to QM-MM optimisation using the QSite module and the DFT-B3LYP method together with the OPLC_2005 force field. Distance dependant dielectrics were applied. Finally, the docking centroid were selected to include an area of $10 \AA$ from the MEEVD peptide. The peptides were sketched at $\mathrm{pH} 7,0$ and tautomers were generated using the LigPrep module. Conformations were generated using the ConfGen module. Peptides were docked using the Glide module with the OPLS3e force field and partial charges were applied. Flexible ligand sampling, a soften potential scaling factor of 0,8 and a partial charge cut-off of 0,15 were used. Finally, the docked ligands were subjected to a binding energy calculation using the Prime MM-GBA module, the OPLS3e force field and VGBD solvation model. The Glide Score, and MMGBSA dG Bind (binding energy in kcal.mol$\left.{ }^{1}\right)$ is reported.

(1) Marklund, E. G.; Degiacomi, M. T.; Robinson, C. V.; Baldwin, A. J.; Benesch, J. L. P. Structure 2015, 23, 791-799.

(2) Fontana, J.; Fulton, D.; Chen, Y.; Fairchild, T. A.; McCabe, T. J.; Fujita, N.; Tsuruo, T.; Sessa, W. C. Circ. Res. 2002, 90, 866-873.

(3) Van Der Spuy, J.; Kana, B. D.; Dirr, H. W.; Blatch, G. L. Biochem. J. 2000, 345, 645651.

(4) Blatch, G. L.; Lässle, M.; Zetter, B. R.; Kundra, V. Gene 1997, 194, 277-282.

(5) Edkins, A. L.; Ludewig, M. H.; Blatch, G. L. Int. J. Biochem. Cell Biol. 2004, 36, 15851598.

(6) Hunter, M. C.; O’Hagan, K. L.; Kenyon, A.; Dhanani, K. C. H.; Prinsloo, E.; Edkins, A. L. PLoS One 2014, 9, e86842.

(7) Schrödinger Release 2019-2; Maestro, Schrödinger, LLC: New York, NY, 2019.

(8) Scheufler, C.; Brinker, A.; Bourenkov, G.; Pegoraro, S.; Moroder, L.; Bartunik, H.; Hartl, F. U.; Moarefi, I. Cell 2000, 101, 199-210. 